\title{
Lernen von Shoppingcentern
}

\author{
Perspektiven für die innerstädtische \\ Einzelhandelsentwicklung in Klein- und Mittelstädten
}

\author{
Tanja Korzer
}

Online publiziert: 24. Juni 2015

(C) Die Autor(en) 2015. Dieser Artikel ist auf Springerlink.com mit Open Access verfügbar.

Zusammenfassung Shoppingcenter stehen in der Kritik, die Einzelhandelslandschaft der Innenstädte in Bezug auf das Einzelhandelsangebot und die geschaffenen Raumsituationen (vor allem in der Mall) zu uniformieren. Doch was machen Projektentwickler und Betreiber von Shoppingcentern u. a. bei der strategischen und konzeptionellen Gestaltung (z. B. in Bezug auf Kundenansprache, Branchen- und Sortimentsmix) richtig? Was können die Akteure in Klein- und Mittelstädten von ihnen für die innerstädtische Einzelhandelsentwicklung in Klein- und Mittelstädten lernen? Denn die Umsetzung geschlossener Shoppingcenter-Komplexe ist in der meist kleinteiligen Einzelhandelslandschaft dieses Stadttypus nur selten möglich und wünschenswert. Hinzu kommt, dass eine große Anzahl von Klein-und Mittelstädten von Schrumpfungstendenzen betroffen ist bzw. zukünftig betroffen sein wird, was maßgeblich Einfluss auf die Entwicklungsperspektiven des innerstädtischen Einzelhandels hat.

Aus diesen Gründen sind alternative Lösungsansätze für die Zusammenarbeit von Akteuren der Stadtentwicklungsplanung sowie lokalen Einzelhändlern in Klein- und Mittelstädten gefragt. Vor diesem Hintergrund erfolgte basierend auf einer interdisziplinär angelegten Literatur- und Dokumentenanalyse, unterschiedlichen Sekundärdatenauswertungen und Primärdatenerhebungen die Identifizierung von Shoppingcenter-Erfolgsfaktoren. Deren inhaltliche Systematisierung führte im Weiteren zur Formulierung eines übertragbaren Zielkatalogs für die innerstädtische Einzelhandelsentwicklung in Klein- und Mittelstädten. Die Anwendung des Zielkatalogs und dessen Reflektion in der Praxis erfolgte in Torgau (Sachsen) und zeigte wesentliche exemplarische Handlungsdefizite auf.

Der folgende Beitrag umreißt in Kurzform die Ergebnisse der Dissertation zu diesem Thema, wobei es in diesem Beitrag vor allem darum geht, die Vorgehensweise

T. Korzer $(\bowtie)$

Institut für Stadtentwicklung und Bauwirtschaft (ISB), Universität Leipzig,

Leipzig, Deutschland

E-Mail: korzer@wifa.uni-leipzig.de 
bei der Übertragung von Shoppingcenter-Erfolgsfaktoren aus verschiedenen Fachperspektiven (Architektur, Betriebswirtschaft etc.) auf die Stadtentwicklungsplanung mit dem Fokus auf die Innenstädte von Klein- und Mittelstädten darzustellen.

\title{
Schlüsselwörter Klein- und Mittelstädte $\cdot$ Shoppingcenter $\cdot$ Stadtentwicklung ·
} Handel

\begin{abstract}
Towns are regional economic and employment centres and hence play a key role in Germany's economic, structural and social development. After all, "over half the population of Germany, around $54 \%$, now lives in towns, and the majority of dependent employees work there." (Gatzweiler, Starke Mittelstädte: Städtebauförderung in ländlichen Räumen, Kongress 22. Juni 2010 in Berlin, Kongressdokumentation S. 17-23, 2010) About $50.6 \%$ of all jobs in Germany are located in towns, while their inhabitants account for $54.6 \%$ of the country's purchasing power. Accordingly, they are an essential component of German's spatial structure which, in line with its decentralized concentration, is divided into growth cores of different sizes and significance.
\end{abstract}

Being aware of this, in recent years German regional planning authorities have paid more attention to the internal development of urban areas and also the urban development of towns and small cities by revising their funding programmes (e.g. by promoting active town and borough centres as well as small towns and municipalities). Regarding the specific task of town-centre retail development, alongside 'traditional' (i.e. formal) planning instruments such as zoning and land use planning, informal yet practical planning instruments like retail location and centre strategies are increasingly being applied. However, the resulting strategies usually address the urban area as a whole. Only rarely do urban development planning authorities draw up concrete town-centre plans (e.g. in connection with strategic planning such as master planning) combined with marketing proposals for areas with precise boundaries. Moreover, translating planning intentions into concrete schemes is often impeded by a lack of both financial resources and expertise.

Furthermore, towns (especially in eastern Germany) are beset by shrinkage tendencies. In these areas, the already straitened municipal finances are exacerbated by:

- Fluctuating trade tax revenue due to the economy leading to local government deficit,

- Rising spending, especially in the social sphere,

- Growing municipal debt.

Given this, it is interesting to note that a survey conducted among all large towns and small cities in Germany revealed that above-average importance is attributed to retail in connection with urban development.

The retail landscape in towns is characterized by some specific features, such as the dominance of medium-sized, often owner-managed shops-whose popularity with customers is, however, dwindling. Other shortcomings include:

- An unbalanced range with a tendency towards substandard items, in particular in clothing and consumer electronics

- The absence of certain goods, especially at supermarkets 
- The absence of certain types of shops (e.g. attractive chain stores)

- High vacancy rates

- No standards opening times

These weaknesses are partly a reflection of the declining financial strength of medium-sized retailers in towns and small cities, the majority of whom are unable to fully withstand the sweeping changes taking place such as population decline, increasing competitive pressure and customers' changing behaviour.

Judging by the current environment, there is an urgent need for alternative action by local authorities to support local retailers in order to stabilize urban development in towns in Germany. On the one hand, it's a question of safeguarding public services, i.e. providing the general public with basic institutions as well as retail, social and technical infrastructure services in as much of the country as possible. On the other hand, the increasing competition for people, jobs and investment means that towns are under pressure to improve or at least maintain their attractiveness. Town centres have an important part to play in this respect since they are the crystallization points of urban functions. Retail, an essential part of urban development, can help make town centres more attractive and stabilize them.

But what strategies in the world of retail can lead to an improvement of the situation described? The currently successful format of the shopping centre provides some interesting pointers. Since their introduction in the USA, the development of shopping malls has been an economic success. The innovation of shopping centres boils down to their ability to identify the main qualities of urban spatial and functional networks and to implement them in a closed system, along with the introduction of a system of centre management handling the central organization of all the aspects of running a shopping centre.

But not every town or small city makes a suitable location for a shopping centre. The growing focus on town centres has presented developers and local authorities with new challenges. The format of the traditional shopping centre (with a sales area of at least $10,000 \mathrm{sq} \mathrm{m}$ ) can't usually be transplanted to town centres in an economically successful manner without upsetting urban development or local functions. The main reasons are the mostly small units available and aspects of the retail environment such as centrality, purchasing power, and the density and development of the population.

Consequently, alternative solutions are required. Back in 1998 Ulrich HATZFELD stated: "What is far more interesting than the question of how to counteract the impact of malls in Germany ... is to examine whether the success of the mall principle contains any pointers for urban planning strategies. Although a simulation of a simulation is out of the question, confronting the contradictory sometimes gets us further than continuing to pursue "tried-and-trusted modes of thought'." (Hatzfeld, Kunstwelten. Künstliche Erlebniswelten und Planung, Informationskreis für Raumplanung e. V., 1998). Adopting this approach, the author of this paper raises the following hypothesis:

In order to promote the economically viable and sustainable development of the high street and town centres, we can learn something from shopping centres.

To support this hypothesis, an extensive set of shopping centre success factors was identified from the literature based on my own empirical surveys and secondary statistical data. These success factors were divided into four overarching categories: 
Table: Success factors of shopping centres

\begin{tabular}{ll}
\hline $\begin{array}{l}\text { Planning and layout } \\
\text { Integration into the urban environment, architec- } \\
\text { ture, functional networking }\end{array}$ & $\begin{array}{l}\text { Retail and marketing } \\
\text { Adaptation to target groups } \\
\text { Makeover of the development zone }\end{array}$ \\
$\begin{array}{l}\text { Networking and steering } \\
\text { Coordinated retail }\end{array}$ & $\begin{array}{l}\text { Observation (Monitoring) } \\
\text { Coordinated marketing and management }\end{array}$ \\
\hline
\end{tabular}

To harness these success factors on the high street, the overarching goals and action necessary to bring about sustainable, economically viable town-centre retail development were derived. The set of aims was then applied to the example of Torgau, a town in Saxony using the procedure described below.

Whether these findings can be generalized and transferred was assessed by applying these aims to Torgau. In the first step, the defined overarching aims were operationalized at the local level, and the strengths and weaknesses were analysed on the basis of the evaluation criteria identified. The second step aided the development of an idealized strategy for town-centre retail and discussion of it with public and private stakeholders.

This approach showed that the set of aims can indeed be translated into practice in a manner which is beneficial for urban development planning. The transfer of the goals and their operationalization provided a substantial share of the ideas for the development of town-centre planning and also highlighted weak spots of possible implementation in the four categories as outlined below:

- Planning and layout:

- Failure to fully grasp and exploit the quality of the town-planning, architectural and functional aspects of a historically evolved town centre as a USP for the retail function.

- The almost non-existent possibility to develop new retail units owing to the dense nature of the built-up areas.

- Insufficient financial resources for necessary investment in public spaces.

- Retail and marketing:

- The lack of agreement and organization of the actors involved leads to various problems.

- Developing a coordinated retail offering encouraging synergies requires a high level of liaison and management between retailers.

- Networking and steering

- The mutual expectations of the local authority and the retailers make it difficult to establish a system of neutral, centrally geared town-centre coordination.

- The high number of individual landlords in a wide range of financial positions hampers concerted action.

- Obtaining financial support for urban development funding is increasingly difficult due to the declining levels of funding available and a lack of human resources at the local authority.

- Observation

- Constant monitoring ties up a substantial number of personnel.

Even if the weaknesses mentioned pose a major challenge for the development of town-centre retail, the test results show that the key to the solution lies above all in persuading stakeholders of the need to work together and supporting coordination to encourage renewal within urban development. 
The set of tools developed is mainly aimed at the local authorities of towns and small cities. They are designed to strengthen awareness of the importance of integrated planning and to regard towns and cities as both built-up areas and functional networks with important potential for future developments.

Keywords small and medium sized cities - Shopping Mall · urban development · retail

\section{Aktuelle Situation der Stadtentwicklung in Klein-und Mittelstädten und deren Bedeutung innerhalb des deutschen Städtesystems}

Klein- und Mittelstädte ${ }^{1}$ bestimmen als regionale Wirtschafts- und Arbeitsmarktzentren die wirtschaftliche, raumstrukturelle und gesellschaftliche Entwicklung Deutschlands entscheidend mit, denn ,mehr als die Hälfte der Bevölkerung in Deutschland, rund 54\%, lebt heute in Klein- und Mittelstädten, die Mehrheit aller abhängig Beschäftigten arbeitet dort" (Gatzweiler 2010, S. 17). Aktuell befinden sich noch rund 50,6\% aller Arbeitsplätze in Klein- und Mittelstädten. 54,6\% der Kaufkraft entfallen auf die Bewohner dieses Stadttyps (Adam 2010, Datenstand 2008). Das bedeutet auch, dass Städte und Gemeinden mit bis zu 100.000 Einwohnern den größten Anteil an Umsatz (60\% des gesamten Einzelhandelsumsatzes) und Verkaufsfläche (65\% der Gesamtverkaufsfläche des Einzelhandels) aufweisen. ${ }^{2}$ Dementsprechend sind sie ein wesentlicher Baustein des innerdeutschen Raumordnungsgefüges, welches, dem Leitmotiv der dezentralen Konzentration folgend, in Wachstumskerne unterschiedlicher Größe und Bedeutung gegliedert ist.

Im Bewusstsein dessen hat die Raumordnungsplanung in den vergangenen Jahren einerseits die Innenentwicklung von Städten und andererseits die Stadtentwicklung von Klein- und Mittelstädten durch Neuauflage von Förderprogrammen (wie z. B. aktive Stadt- und Ortsteilzentren sowie kleinere Städte und Gemeinden) in den Fokus ihres Interesses gerückt. Darüber hinaus finden in Klein- und Mittelstädten mit Blick auf die spezifische Aufgabe der innerstädtischen Einzelhandelsentwicklung neben den „klassischen“, formell verankerten Planungsinstrumenten wie z. B. der Bauleitund Flächennutzungsplanung zunehmend handlungsbezogene, informelle Planungsinstrumente wie z. B. Einzelhandelsstandort- und Zentrenkonzepte, Anwendung (Hirsch 2011, S. 18). ${ }^{3}$ Allerdings beziehen sich die damit formulierten strategischen Aussagen i. d. R auf die Gesamtstadt. Die Entwicklung einer konkreten (möglichst grundstücksscharfen) Innenstadtkonzeption (z. B. im Rahmen einer strategischen Planaussage wie einer Masterplanung), verbunden mit einer Marketingstrategie, wird dagegen zu selten als Aufgabe der Stadtentwicklungsplanung erkannt und umgesetzt.

\footnotetext{
${ }^{1}$ Der vorliegende Beitrag folgt der Definition der Klein- und Mittelstund gemme der Stadtforschung auf Bundesebene (wie z. B. die laufende Raumbeobachtung des BBSR, wonach es sich bei StSi Sn mit 5000 bis 20.000 Einwohnern um Kleinstädte und mit 20.000 bis 100.000 Einwohnern um Mittelstrn u handelt.

${ }^{2}$ GfK GeoMarketing zitiert nach Deutsche Hypothekenbank/Immobilien Research 2009, S. 14; 20. Allerdings kann in Großstädten eine höhere Flächenproduktivität festgestellt werden.

${ }^{3} \mathrm{Zu}$ bemerken ist dabei, dass Verantwortliche der Wirtschaftsförderung dem Einzelhandel eine größere Relevanz zuschreiben, als Verantwortliche der Stadtplanung.
} 
Zudem fehlt es häufig an personellem Know-how und finanziellem Spielraum, mögliche planerische Ansätze in konkrete Maßnahmen zu übersetzen.

Darüber hinaus sind vor allem Klein- und Mittelstädte in Ostdeutschland von Schrumpfungstendenzen betroffen. In diesen Kommunen verschärft sich die generell als problematisch eingeschätzte Situation des kommunalen Finanzsystems, welche vor allem durch: ${ }^{4}$

- schwankende konjunkturabhängige Gewerbesteuereinnahmen und dem daraus resultierenden kommunalen Finanzierungsdefizit,

- steigende Ausgaben, vor allem im sozialen Bereich und

- steigende Verschuldung der Kommunen

geprägt sind.

Vor diesem Hintergrund ist es interessant, dass eine Befragung aller deutschen Mittelstädte ${ }^{5}$ ergab, dass dem Einzelhandel eine überdurchschnittlich hohe Bedeutung für die Stadtentwicklung beigemessen wird. Konkrete Erwartungen bestehen vor allem hinsichtlich ,einer funktionalen Stärkung der Innenstadt (93,1\%), der Stärkung der Einzelhandelszentralität (70,6\%) sowie der Verbesserung der Versorgungssituation für die Stadtbewohner (61,8\%)" (Hirsch 2011, S. 18).

Die Einzelhandelslandschaft in Klein- und Mittelstädten ist allerdings durch einige spezifische Besonderheiten geprägt, wie bspw. die Dominanz mittelständischer, häufig inhabergeführter Einzelhandelsunternehmen, welche zunehmend weniger vom Kunden als Einkaufsalternative akzeptiert werden. Darüber hinaus lassen sich weitere Defizite benennen (Hirsch 2011, S. 21):

- unausgewogene Sortimentszusammensetzung mit Tendenz zu minderwertigen Angeboten, vor allem ein unzureichendes Angebot im Bereich Textilien und Unterhaltungselektronik,

- Lücken in der Nahversorgung, vor allem in Bezug auf das Angebot von Vollsortimentern,

- Fehlen bestimmter (Einzelhandels-)Betriebsformen und ihre entsprechenden Einzelhandelsangebote (z. B. attraktive Filialisten),

- hohe Leerstände,

- uneinheitliche Öffnungszeiten.

Ein Großteil dieser Schwächen ist u. a. Ausdruck der sinkenden Finanzkraft mittelständischer Einzelhändler, so dass nur noch bedingt Anpassungen an die sich vollziehenden umfassenden Veränderungen (wie z. B. Bevölkerungsrückgang, wachsendem Konkurrenzdruck, verändertem Kundenverhalten) erfolgen.

\section{Problemstellung und Untersuchungsansatz}

Die aktuellen Rahmenbedingungen verdeutlichen, dass der Bedarf an alternativen Ansätzen zur Unterstützung des lokalen Einzelhandels seitens der Stadtverwaltung

\footnotetext{
${ }^{4}$ Vgl. Bundesfinanzministerium, Ergebnisse der Gemeindefinanzkommission des Bundes, 2012.

${ }^{5}$ Vgl. Hirsch 2011: Im Rahmen einer von der IREBS durchgeführten Studie zur Einzelhandelssituation in Mittelstädten wurden alle deutschen Mittelstädte zwischen 50.000 und 250.000 EW befragt. 
zur Stabilisierung von Stadtentwicklungsprozessen in Klein- und Mittelstädten in Deutschland groß ist $^{6}$. Einerseits geht es um die Absicherung der öffentlichen Daseinsvorsorge, d. h. um die möglichst flächendeckende Grundversorgung der Bevölkerung mit Einrichtungen und Dienstleistungen der einzelhändlerischen, sozialen und technischen Infrastruktur. Andererseits erfordert der steigende Wettbewerbsdruck um Bevölkerung, Arbeitsplätze sowie Investitionen die Erhaltung bzw. Steigerung der Attraktivität der Städte. Sowohl den Innenstädten als Kristallisationspunkte städtischer Funktionen im Allgemeinen als auch dem innerstädtischen Einzelhandel als wesentlicher Teil der Stadtentwicklung im Speziellen kommt dabei eine besondere Bedeutung zu.

Doch welche Strategien aus dem Themenfeld des Einzelhandels können zu einer Verbesserung der beschriebenen Situation führen? Diesbezüglich bietet das aktuell erfolgreich agierende Einzelhandelsformat des Shoppingcenters ${ }^{7}$ interessante Denkansätze, denn seit seiner Entstehung in den USA kann die Shoppingcenter-Entwicklung aus betriebswirtschaftlicher Sicht als Erfolgsgeschichte beschrieben werden ${ }^{8}$. Kern der Innovation Shoppingcenter ist einerseits das Erkennen des Zusammenspiels zwischen Raumverflechtungen und Einzelhandelsangeboten, deren Umsetzung in einem funktional geschlossenen System sowie andererseits die Etablierung eines Centermanagements, welches alle Belange des Shoppingcenter-Betriebs zentral organisiert.

Auch in Deutschland entstanden nach Angaben des EHI Retail Institut bis 2014 460 Shoppingcenter mit einer Verkaufsfläche von $10000 \mathrm{~m}^{2}{ }^{9}$ Sowohl in Mittel- als auch in Großstädten findet bis heute eine kontinuierliche Shoppingcenter-Entwicklung statt, wobei vor allem Innenstadtzentren im Fokus der Entwicklung stehen (Ortner 2014, S. 148-161) (Just 2014, S. 34-48 und Falk 2014, S. 105-117).

Doch nicht jede Klein- und Mittelstadt eignet sich als Standort für ein Shoppingcenter. Besonders die zunehmende Innenstadtorientierung stellt Centerentwickler und kommunale Vertreter vor neue Herausforderungen. Denn i. d. R ist das Format des klassischen Shoppingcenters (mit einer Verkaufsfläche ab ca. $10000 \mathrm{~m}^{2}$ ) nicht ohne weiteres städtebaulich, funktional verträglich sowie wirtschaftlich erfolgreich auf Klein- und Mittelstädte übertragbar. ${ }^{10}$ Wesentliche Ursache dessen sind die innerstädtisch meist kleinteiligen Flächenzuschnitte und -dimensionen im Stadt-

\footnotetext{
${ }^{6}$ Beispielsweise greift das Förderprogramm „Aktive Stadt- und Ortsteilzentren“ (Start 2008) als Instrument der nationalen Stadtentwicklungspolitik die Handlungsfelder der NSP auf und formuliert weitere Zielvorgaben wie z. B. die Funktionsvielfalt und Versorgungssicherheit, die Aufwertung des öffentlichen Raumes, die Stadtbaukultur sowie die partnerschaftliche Zusammenarbeit.

${ }^{7}$ Zur Definition vgl. Urban Land Insitut 1993, S. 4.

${ }^{8}$ Bezüge zu Passagen und Warenhäusern werden aufgrund der weiteren Differenzierung bewusst nicht hergestellt.

${ }^{9}$ http://www.handelsdaten.de/themen/297/shopping-center-de/, abgerufen am 29.08.2014. Bezogen auf das Jahr 2014 existierten laut EHI Retail Institut 460 Shoppingcenter mit einer Gesamtmietfläche von ca. 14,5 Mio. $\mathrm{m}^{2}$.

${ }^{10}$ Die Erfahrungen mit Centerprojekten aus einem großstädtischen Kontext sind nicht ohne Weiteres auf Klein- und Mittelstädte zu übertragen, denn dieser Stadttypus ist durch eine hohe Heterogenität in Bezug auf wirtschaftliche, demografische und funktionelle Rahmenbedingungen [vgl. Kabisch et al. 2004, S. 32; Baumgart 2004, S. 7] sowie die historische Entwicklung, die Lagesituation und die Bebauungsstrukturen (vorwiegend kleinteilig, häufig gut erhaltene historische Bausubstanz) geprägt. Vielmehr ist „Ihre Vielfalt (ist) typisch für die deutsche Klein- und Mittelstadt [Flacke 2004, S. 27].“
} 
zentrum, handelswirtschaftliche und funktionale Rahmenbedingungen, wie z. B. Zentralität, Kundendichte, Kaufkraft, Bevölkerungsdichte und -entwicklung sowie Erreichbarkeit.

Aus diesem Grund bedarf es alternativer Lösungsansätze. Bereits 1998 stellte Ulrich HATZFELD fest: ,,Wesentlich interessanter als die Frage, wie man in Deutschland den Auswirkungen von Malls entgegenwirken kann [...], ist eine Auseinandersetzung mit der Frage, ob sich aus dem Erfolg des Mall-Prinzips möglicherweise Ansatzpunkte für Strategien der Stadtplanung ergeben. Dabei kann es keinesfalls um die Simulation der Simulation gehen - aber zuweilen bringt eine Konfrontation mit dem Widersprüchlichen weiter als die Weiterentwicklung ,gesicherter Denkweisen“" (Hatzfeld 1998, S. 50). Entsprechend dieses Denkansatzes stellt die Autorin dieses Beitrages die Hypothese auf:

Es ist möglich, von ausgewählten Shoppingcenter-Erfolgsfaktoren für eine ökonomisch tragfähige und nachhaltige innerstädtische Einzels- und Stadtentwicklung zu lernen (Dabei steht die Benennung möglicher Handlungsansätze für das Zusammenwirken von Stadtentwicklungsplanung und lokalen Einzelhändlern in Klein- und Mittelstädten im Vordergrund).

\section{Analyse der Erfolgsfaktoren von Shoppingcentern}

Die analytische Auseinandersetzung mit dem Untersuchungsgegenstand Shoppingcenter erfolgt aus unterschiedlichen Fach- und Akteursperspektiven, wie bspw. Handelsbetrieb, Städtebau/Architektur, Geografie etc. Dazu wurden Shoppingcenter-Erfolgsfaktoren sowohl durch Literatur- und Dokumentenanalysen als auch Sekundärdatenauswertungen und Primärdatenerhebungen zusammengetragen (vgl. Abb. 1). Der inhaltliche Fokus der Analyse lag dabei vor allem auf dem Spannungsfeld zwischen Stadtentwicklungsplanung und Einzelhandel.

Die Zusammenführung der vielfältigen, sich teilweise überlagernden Erfolgsfaktoren macht es dabei notwendig, eine inhaltliche Systematisierung zu entwerfen, welche in vier Handlungsfeldern mündet. Die Handlungsfelder dienen im Weiteren der Strukturierung des Erfolgsfaktorensets (vgl. Tab. 1) sowie dem daraus abgeleiteten Zielkatalog ${ }^{11}$.

Das Handlungsfeld Gestalten und Verorten beschreibt dabei vor allem städtebauliche, architektonische sowie funktionale Inhalte. Das Handlungsfeld Handeln und Vermarkten ist dagegen durch einen starken Bezug zu möglichen Positionierungsstrategien im Einzelhandel gekennzeichnet. Eine entsprechende inhaltliche und organisatorische Verbindung dieser Schwerpunkte findet dann im Handlungsfeld Vernetzen und Steuern statt. Das Handlungsfeld Beobachten dient abschließend der Absicherung einer fundierten Datenbasis, welche Grundlage strategischer Entscheidungen sein sollte.

\footnotetext{
${ }^{11} \mathrm{Zu}$ beachten ist dabei, dass die inhaltliche Fokussierung und die Reihenfolge der benannten Handlungsfelder aus der Perspektive der Stadtentwicklungsplanung resultieren.
} 


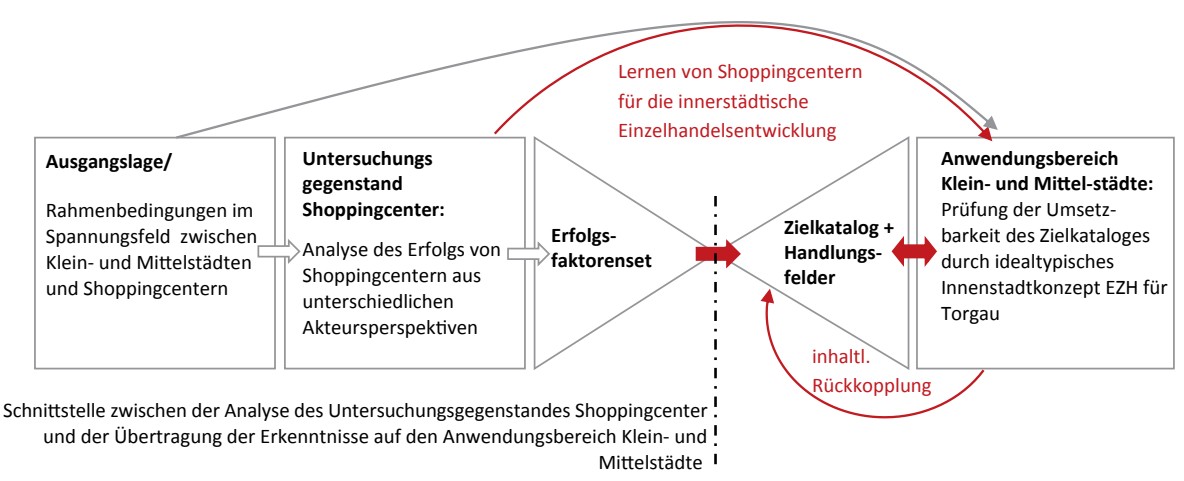

Abb. 1 Vorgehensweise mit Fokus auf die Erfolgsfaktorenanalyse und die Definition eines Zielkatalogs. (eigene Darstellung)

Tab. 1 Erfolgsfaktorenset Shoppingcenter. (eigene Darstellung)

\section{Handlungsfeld Gestalten und Verorten}

Stadträumliche Einbindung/architektonische Gestaltung/funktionale Vernetzung

Standort und damit verbundene stadträumliche sowie funktionale Einbindung, Erreichbarkeit und damit verbundenes Parkplatzangebot

Einheitliche Ausprägung von Strukturmerkmalen wie bspw. Bauweise/Architektur (geschlossenes vs. offenes Shoppingcenter) sowie architektonische Gestaltung (z. B. durch Aufnahme von Raumkanten, Traufhöhen, Fassadengestaltung), Gebäudedimensionierung und Atmosphäre/Ambiente

Flexibilität der Bausubstanz

Lage und Anzahl der Ein- und Ausgänge, Kundenströme

Erzeugung eines Rundlaufs durch Lage der Magnetbetriebe

Gestaltung des Erschließungsraums

Öffentlichkeit versus Privatheit

Bespielung des Erschließungsraums des Shoppingcenters

Handlungsfeld Handeln und Vermarkten

Anpassung an Zielgruppen und Kundenanforderungen

Nutzungskonzept/Funktionsmix

Unterhaltung/Events/Einkaufserlebnis

Service/Personal/Dienstleistung/Convenience

Mieter- und Branchenmix

Attraktivität der Ankermieter

Erreichbarkeit/Parkplatzangebot

Preis-Leistungs-Verhältnis

Erzeugung von Synergie- und Agglomerationseffekten

Verkaufsflächengröße bzw. Geschäftsangebot

Branchen- und Sortimentsmix

Filialisierungsgrad/Ankermieter

Handlungsfeld Vernetzen und Steuern

Zentrales (Center)Management

Einheitliches Geschäftsprinzip

Abgestimmtes Marketing und Management 


\section{Ableitung eines Zielkatalog innerstädtische Einzelhandelsentwicklung für Klein- und Mittelstädte}

Basierend auf dieser inhaltlichen Struktur und den jeweils zugeordneten Erfolgsfaktoren fand die Abstraktion der Aussagen auf der übergeordneten Ebene eines Zielkatalogs statt, welcher auf einen städtischen Kontext wie die Innenstadtentwicklung von Klein- und Mittelstädten übertragbar ist. Der Zielkatalog innerstädtische Einzelhandelsentwicklung verbindet somit die Erkenntnisse der Erfolgsfaktorenanalyse mit den Anforderungen der Stadtentwicklungsplanung. Die Ableitung der einzelnen Ziele wird im Folgenden überblicksartig dargestellt:

\subsection{Handlungsfeld: Gestalten und Verorten}

Das Handlungsfeld Gestalten und Verorten nimmt inhaltlich Bezug auf die übergeordnete Raumordnungsplanung. Insbesondere die im Rahmen der Städtebauförderung formulierten Leitvorstellungen im Bereich des Stadtraums sowie dessen architektonischer Gestaltung wie z. B. Aufwertung des öffentlichen Raums und Sicherung der Stadtbaukultur und den durch die Gesetzgebung verankerten Rahmenbedingungen für Einzelhandelsansiedlungen bilden die Grundlage für die Ableitung des übergeordneten Ziels:

- Entwicklung eines städtebaulich und architektonisch abgestimmten Einzelhandelsstandortes Innenstadt mit engen funktionalen Wechselbezügen.

Erfolgsfaktoren Shoppingcenter: Shoppingcenter werden zunehmend in Innenstädten verwirklicht. Dieser Trend verweist einerseits auf die gezielte planerische Steuerung in Richtung innerstädtischer Handelsentwicklung und ist andererseits ein deutliches Bekenntnis zu den Qualitäten der kompakten, nutzungsgemischten Stadt (z. B. in Bezug auf Architektur, Stadtraum und Urbanität) vor dem Hintergrund sich wandelnder Kundenbedürfnisse.

Das Einzelhandelsformat des Shoppingcenters erfährt in vielen Fällen eine baulichefunktionale Anpassung und wandelt sich so zunehmend von einer suburbanen zu einer urbanen Bautypologie. Die entsprechende Auseinandersetzung mit dem Kontext findet im Wesentlichen in Bezug auf die Flächendimensionierung, die architektonische, städtebauliche und funktionale Integration und Gestaltung statt. Das spiegelt sich vor allem in der Strukturierung der Gebäudekubatur bis hin zur Realisierung offener Shoppingcenter Konzeptionen, in der Öffnung der Fassade oder der Anordnung sowie Anzahl der Ein- und Ausgänge wider. Diese Veränderung zeigt, dass die „urbane Differenz“ des innerstädtischen Standortes sowie die verschiedenen Möglichkeiten der Nutzung und Erzeugung von Synergien zwischen Räumen, Funktionen und Akteuren positiven Einfluss auf die Akzeptanz und damit den Erfolg von Shoppingcentern nehmen können (Siebel 2007, S. 86).

Übertragung auf den städtischen Kontext: Sowohl die Stadtentwicklungsplanung als auch die Projektentwickler/Betreiber bzw. Einzelhändler müssen sich der häufig hohen städtebaulichen urbanen Qualitäten der Innenstädte von Klein- und Mittelstädten zunehmend bewusst werden und sie gemeinsam für diesen Standort weiter- 
entwickeln. Im Unterschied zu einer Shoppingcenter-Planung aus einer Hand stehen dabei die individuelle städtebaulich-bauliche Gestaltung der einzelnen Objekte und deren räumlich-funktionaler Zusammenhang (Ensemblewirkung) im Mittelpunkt des stadtentwicklungsplanerischen Interesses. Daraus leitet sich das Unterziel $1 \mathrm{ab}$ :

\section{- Qualitätssicherung der städtebaulichen, architektonischen und funktionalen Gestaltung des EZH-Standortes Innenstadt (Stichwort: Urbane Differenz).}

Erfolgsfaktoren Shoppingcenter: Das funktionierende Gefüge eines Shoppingcenters ist eng mit der Architektur und der Baustruktur verbunden. Das Gebäude kann dabei durch seine architektonische Qualität als Imageträger fungieren (Brune 2014, S. 142-147).

Als zentrales Organisationsprinzip dient die Gestaltung eines räumlichen wie funktionalen (Erschließungs-)Rundlaufs, welcher durch das Wechselspiel von Ankermietern und ergänzenden Einzelhandelsangeboten sowie Dienstleistungen nach wie vor gekennzeichnet ist. Mit zunehmender Integration eines Shoppingcenters in eine zentrale Handelslage erhöht sich dessen funktionale Vernetzung mit dem städtischen Kontext. Doch unabhängig davon wird die wirtschaftliche Tragfähigkeit u. a. vor allem durch die Aufenthaltsdauer und die erzielten Umsätze beeinflusst.

Übertragung auf den städtischen Kontext: Bezogen auf den städtischen Kontext sollte es somit Ziel sein, einen kohärenten Rundlauf in hochwertiger städtebaulicher und architektonischer Qualität sowie mit einem kontinuierlichen aufeinander abgestimmten Einzelhandelsangebot (Schaffung einer ausgewogenen Balance zwischen Ankermietern, Frequenzbringern [häufig filialisiert] und attraktiven ergänzenden [häufig individuellen] Angeboten zu entwickeln. Dabei ist eine Beurteilung der Passfähigkeit neuer Einzelhandelsangebote (immer im Verhältnis zum städtischen Kontext und zu den kommunalen stadtentwicklungsplanerischen Zielstellungen) in enger Zusammenarbeit zwischen Kommune (Politik/Stadtentwicklungsplanung) und Investor/Projektentwickler/Händler im Sinne der städtebaulichen, architektonischen und funktionalen Qualität eines Projektes erforderlich. Folgende Kriterien sind dabei zu berücksichtigen:

- Aufnahme von Raumkanten sowie Traufhöhen der Umgebungsbebauung,

- Dimensionierung und Strukturierung der Baukörper (in Grund- und Aufriss),

- Fassadengestaltung (Öffnung zum Stadtraum) und Materialität sowie

- Lage der Ein- und Ausgänge.

Daraus resultiert das Unterziel 2:

- Gestaltung eines städtebaulich, stadträumlich und funktional kohärenten „Rundlaufs".

Entscheidendes Verbindungselement zwischen städtischen Funktionen wie bspw. Einzelhandel, Wohnen, Arbeiten ist der häufig über einen längeren Zeitraum gewachsene öffentliche Raum. Er ist Plattform für das, was gemeinhin als „urbanes öffentliches Leben“ bezeichnet wird und er ist Anknüpfungspunkt zu halböffentlichen bzw. privaten Bewegungsräumen z. B. Passagen und Shoppingcentern. 
Erfolgsfaktoren Shoppingcenter: Die Gestaltung des Erschließungsraums des Shoppingcenters folgt einem einheitlichen Entwurf, welcher sich u. a. in aufeinander abgestimmten Materialien, Möblierung, Wasserspielen, Bepflanzungen widerspiegelt. Dessen Belebtheit basiert auf einem möglichst flüssigen Passantenstrom und einer unterstützenden Bespielung durch regelmäßige Events, Marketingaktionen etc. sowie häufig innen liegenden Funktionen z. B. Eiscafés oder temporäre Verkaufsstände.

Übertragung auf den städtischen Kontext: Die künstlich erzeugte Belebtheit im Shoppingcenter ist bereits teilw. Vorbild für eine zusätzliche, ,inszenierte“ Belebung des öffentlichen Raums der Stadt. Die Nutzung dieser Strategie für die Innenstadtentwicklung und den innerstädtischen Einzelhandel kann die Wahrnehmung der Qualitäten des öffentlichen Raums bei seinen Nutzern erhöhen, muss allerdings in Abwägung der übergeordneten Interessen der Kommune, u. a. durch die Erhaltung der Funktion des öffentlichen Raums unter Begrenzung bspw. von Privatisierungstendenzen, erfolgen. Notwendiger Bestandteil dessen ist sowohl die hochwertige Gestaltung des Stadtraums (z. B. durch Stadtmöblierung, Materialität des Straßenbelags, Beleuchtung) als auch die Attraktivierung der Schnittstellen zwischen öffentlichen und privaten Räumen wie z. B. Schaufenster, Ladeneingänge. Dazu ist ein koordiniertes Vorgehen unter aktiver Einbeziehung der Akteure des Stadtmarketings, des Stadtmanagements, der Stadtentwicklungsplanung sowie des Einzelhandels und der Immobilieneigentümer notwendig. Daraus leitet sich das Unterziel 3 ab:

- Gestaltung und Aufwertung des öffentlichen Raums sowie Erhaltung seiner öffentlichen Funktion.

\subsection{Handlungsfeld: Handeln und Vermarkten}

Erfolgsfaktoren Shoppingcenter: Sowohl auf der Nachfrage- als auch auf der Angebotsseite vollziehen sich umfassende Veränderungen. Einerseits müssen sich der Einzelhandel und damit auch die Shoppingcenter einem immer stärker differenzierten Konsumentenverhalten stellen. Zudem begründen sinkende Flächenproduktivitäten und fortschreitende Konzentrationsprozesse im Einzelhandel unterschiedliche Anpassungsstrategien der Unternehmen.

Übertragung auf den städtischen Kontext: Aus Sicht der Stadtentwicklungspolitik steht dagegen die Absicherung der Funktionsvielfalt und Versorgungssicherheit der Bevölkerung im Mittelpunkt des Interesses. Das Handlungsfeld Handeln und Vermarkten verbindet diese beiden Perspektiven und formuliert übergeordnete Zielstellungen, welche die Entwicklung der Gesamtagglomeration der innerstädtischen Einzelhandelslage fokussiert. 
Das übergeordnete Ziel im Handlungsfeld Handeln und Vermarkten lautet:

\section{- Anpassung des innerstädtischen Einzelhandelsangebotes an veränderte Kundenbedürfnisse und Sicherung einer größtmöglichen Angebotsvielfalt und -tiefe mit Blick auf die Gesamtagglomeration Innenstadt.}

Erfolgsfaktoren Shoppingcenter: Die Zusammensetzung und Charakterisierung zukünftiger Kundengruppen wird maßgeblich durch den sich vollziehenden demografischen Wandel, der vor allem durch sinkende Bevölkerungszahlen sowie das Steigen des Durchschnittsalters gekennzeichnet ist, beeinflusst. Eng damit verbunden sind die spürbaren Veränderungen der Kundenbedürfnisse. So ist zu beobachten, dass vor allem der Wunsch nach Convenience-, Service- und Erlebnisangeboten, bei gleichzeitig stark ausgeprägtem Preis-Leistungs-Bewusstsein steigt (Stichwort „smart shopping“" (Bastian 1999, S. 2; Besemer 2004, S. 64; Bolz 2002, S. 111; Heinritz et al. 2003, S. 155; Koprian 2014, S. 137-141; Purper 2007, S. 14; Sturm 2006, S. 97) (Purper 2007, S. 14) ${ }^{12}$. Das spiegelt sich auch in den wesentlichen Entscheidungskriterien zur Wahl der Einkaufsstätte durch den Konsumenten wider (vgl. Abb. 2).

Das Modell nach SCHMITZ/KÖLZER (vgl. Abb. 2) gliedert die kaufrelevanten Zusammenhänge in zwei übergeordnete Einflussbereiche - beobachtbare und nicht-beobachtbare Determinanten des Kaufverhaltens, wobei der entscheidende Steuerungsspielraum in der Erfüllung der Kundenanforderungen an die Einkaufsstätte liegt. Dabei ist es von Bedeutung, dass Kunden sowohl das innerstädtische Einzelhandelsangebot als auch das des Shoppingcenters, als Agglomeration in seiner Gesamtheit wahrnehmen ${ }^{13}$, bewerten und basierend darauf ihre Kaufentscheidungen treffen (z. B. in Bezug auf das gefühlte Preis-Leistungs-Verhältnis) (Germelmann und Neder 2012, S. 339 f.).

Die Betriebsform Shoppingcenter hat die Möglichkeit, differenziert und zielgerichtet auf die sich dynamisch verändernden Kundenanforderungen zu reagieren. Dazu gehören bspw.

- die verstärkte Inszenierung des Einkaufens als Erlebnis, die kontinuierliche Überprüfung und Anpassung des Mieter- und Branchenmixes sowie die gezielte Auswahl und Attraktivität der Ankermieter. Entscheidend ist dabei die ausgewogene Mischung der Angebote (sowohl internationale und nationale Filialisten als auch lokale individuelle Einzelhändler bzw. lokale Filialisten),

- eine gute Erreichbarkeit, entweder durch den ÖPNV und/oder durch die direkte Anbindung der Einzelhandelsflächen an ein i. d. R großflächiges, meist kostenloses bzw. kostengünstiges Parkangebot,

- einheitliche Öffnungszeiten sowie

\footnotetext{
${ }^{12}$ „Der Begriff ,Smart Shopping ${ }^{6}$ hat seinen Ursprung in den USA. Wörtlich übersetzt bedeutet Smart Shopping in etwa schlau oder geschickt einkaufen; häufig wird auch vom ,cleveren' Einkaufsverhalten gesprochen." (Esser 2002, S. 11; vgl. auch Much r und Stein 2008, S. 5 f.).

${ }^{13}$ HANSEN bezieht diese Feststellung bspw. auf Betriebsformen und Kooperations- sowie Integrationsformen filialisierter Unternehmen (vgl. Hansen 1990, S. 111). Ein ähnlicher Denkansatz wird bei vergleichenden Untersuchungen zwischen Internet-Handel und Shoppingcenter sowie dem stationären Handel angewendet (vgl. Dach 2002; Wengler 2005).
} 


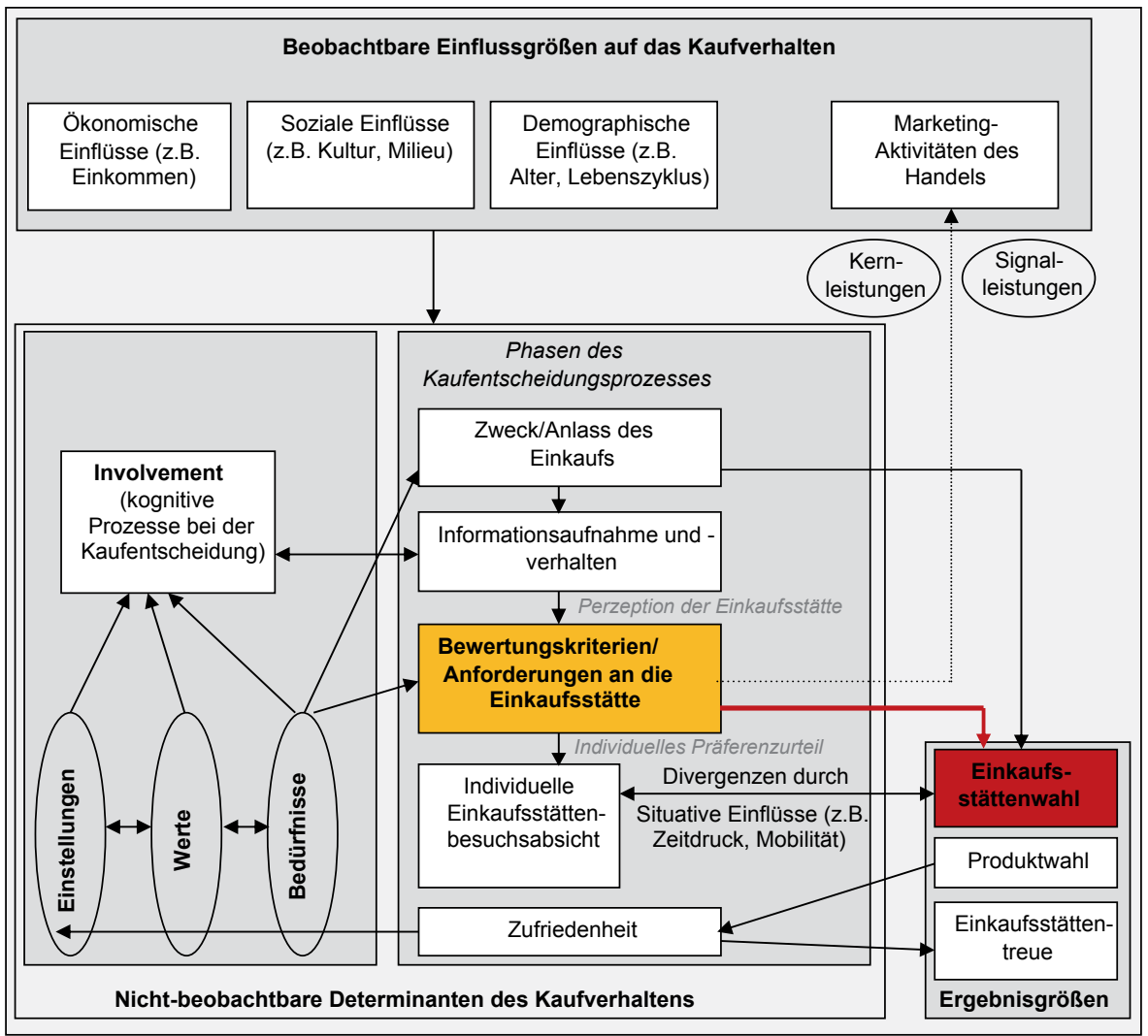

Abb. 2 Gegenüberstellung der Kriterien des Kundenwahlverhaltens und der betriebswirtschaftlich orientierten Erfolgsfaktoren von Shoppingcentern. (eigene Darstellung)

- eine attraktive Einkaufsatmosphäre sowohl durch die architektonische Gestaltung als auch die Bespielung der Mallflächen (Entwicklung zum Third Place) (Mikunda 2009).

Übertragung auf den städtischen Kontext: Bezogen auf den Standort Innenstadt bedeutet das für die lokalen (überwiegend mittelständischen) Einzelhändler, sich klar zu positionieren und ihr Profil zu stärken. Zentraler Ansatzpunkt dafür sollte nach FUNCK/GUTKNECHT/STUMPF das Geschäfts- bzw. Einkaufsstättenimage sein (das Image ist sowohl Bestandteil der Faktoren zur Wahl der Einkaufsstätte als auch der betriebswirtschaftlich orientierten Erfolgsfaktoren von Shoppingcentern). „Die Positionierung stellt durch die Beeinflussung des Images (Imagepolitik) besonders für den mittelständischen Einzelhandel ein wesentliches Instrument zur bewussten Abhebung und Individualisierung der jeweiligen Betriebsstätte dar" (Funck et al. 2007, S. 32). Folgende Elemente sind dabei von besonderer Bedeutung (Funck et al. 2007, S. 33):

- Bestimmung der anzusprechenden Zielgruppe,

- Definition von Verkaufsargumenten (im Sinne des Nutzens für den Konsumenten), 
- eindeutige Preispositionierung in Verbindung mit klarer Preiskommunikation,

- Vermittlung von Werten bezüglich der angebotenen Ware.

Daraus ist das Unterziel 1 abzuleiten:

\section{- Anpassung und Abstimmung des innerstädtischen Einzelhandelsangebotes an steigende Convenience-, Service- und Erlebnisbedürfnisse.}

Erfolgsfaktoren Shoppingcenter: Die Verkaufsflächengröße ist ein entscheidender Erfolgsfaktor von Shoppingcentern, denn ab ca. 15,000 $\mathrm{m}^{2}$ können ein umfassender Branchenmix (mit einer hinreichend großen Anzahl von Fachgeschäften und Filialisten/Ankern) abgebildet und damit größtmögliche Synergien zwischen den Einzelhandelsangeboten erzeugt werden. ${ }^{14}$ Auf den städtischen Kontext übertragen bedeutet das im Umkehrschluss, je vollständiger der angebotene, räumlich kontinuierlich zusammenhängende Branchen- und Sortimentsmix innerhalb einer innerstädtischen Einzelhandelslage, desto attraktiver und anziehender wirkt der Standort Innenstadt auf den Kunden. MONHEIM stellt in diesem Zusammenhang über einen Vergleich von Einzelhandelslagen z. B. in Nürnberg und Leipzig fest, dass die Stadtbesucher bei einem höheren Geschäftsbesatz auch durchschnittlich mehr Geschäfte besuchen (Mohnheim 2011, S. 25 f.).

Ein wesentlicher Vorteil des Shoppingcenter Betriebs ist zudem die Steuerung und gezielte Gestaltung des Branchen- und Mietermixes im Center. Ein diesbezüglich wichtiges Steuerungsinstrumentarium ist die Möglichkeit der Vereinbarung einer individuellen Umsatzmiete; wobei die Grundmiete für jeden Mieter in gleicher Höhe erhoben wird und der über die Grundmiete hinausgehende Mietanteil individuell, entsprechend des Umsatzes, dynamisch festgelegt werden kann (Martin 2003, S. 156). ${ }^{15}$ So ist es möglich, auch kleine umsatzschwächere Mieter, mit einem für den Branchen- und Mietermix interessanten Einzelhandels- oder Serviceangebot, im Center anzusiedeln. Die aktuell wachsende Konkurrenz durch den Onlinehandel und die damit verbundene erschwerte räumliche Zuordnung des getätigten Umsatzes in den Distributionskanälen führt derzeit zu Diskussionen über die künftige Berechnung der Mieten im Shoppingcenter.

Darüber hinaus wird der Mietflächenbesatz im Shoppingcenter an der möglichen Erzeugung synergetischer Nachbarschaften zwischen einzelnen, dafür geeigneten Mietern ausgerichtet. Das bedeutet, dass durch die gezielte Koordinierung der einzelnen Standortanforderungen von Einzelhandelsunternehmen (u. a. abhängig von Sortiment, Zielgruppe und Unternehmensstrategie) eine win-win-Situation im Idealfall für möglichst viele Mieter des Shoppingcenters hergestellt werden kann, so dass umfassende Synergie- und Agglomerationseffekte erzeugt und genutzt werden können. Die Praxiserfahrung zeigt, dass sich verschiedene Branchenanordnungen bzw.

\footnotetext{
${ }^{14}$ Basierend auf Interviews mit: Matthias Böning, mfi Management für Immobilien AG; Andrej Pomtow, MULTI Development Germany GmbH; Thomas Doerr, CENTRUM/COMFORT Center Consulting $\mathrm{GmbH}$; Andreas Fuchs, ECE Projektmanagement G.m.b.H und Co. KG; Dr. Björn Isenhöfer, CONCEPTA Projektentwicklung GmbH.

${ }^{15}$ Gespräch am 23.03.2011 mit Jörg Waldrich, Center Manager Reschop Carré, Hattingen.
} 
Clusterungen bewährt haben, wie z. B. Drogerien und großflächiger Lebensmitteleinzelhandel im Basement, verschiedene Textilanbieter im EG oder OG.

Um diese Angebotszusammenhänge möglichst nicht zu unterbrechen, ist es notwendig, Leerstände im Shoppingcenter zu vermeiden. Die bekannten ,Sockengeschäfte" sorgen im Fall einer notwendigen zeitlichen Überbrückung zwischen Vor- und Nachmieter für die Kontinuität des vielfältigen Angebotes in der Kundenwahrnehmung (Vermeidung von dunklen Schaufenstern).

Übertragung auf den städtischen Kontext: Auch am Einzelhandelsstandort Innenstadt kann die gezielte Ergänzung von Einzelhandelsangeboten, die Erzeugung von Synergien und das Management von Leerständen entscheidend zum Erfolg des Standortes beitragen. Zwischen folgenden Sortimenten innerhalb einer Branche bzw. zwischen folgenden Branchen lassen sich exemplarische Kopplungssynergien benennen:

- Einzelhandelsunternehmen innerhalb der Branche Bekleidung/Wäsche,

- Einzelhandelsunternehmen der Branchen Bekleidung und Schuhe,

- Einzelhandelsbetrieben der Branche Bekleidung/Wäsche und der Branche Sportartikel/Fahrräder/Camping,

- Einzelhandelsunternehmen innerhalb der Branche Nahrungs- und Genussmittel,

- Einzelhandelsunternehmen der Branchen Drogerie/Parfümerie/Apotheke/Sanitätshaus sowie Nahrungs- und Genussmittel,

- Einzelhandelsunternehmen der Branchen Bekleidung/Wäsche sowie Uhren/ Schmuck/Accessoires.

Daraus resultiert folgendes Unterziel 2:

- Erzeugung möglichst großer Synergie- und Agglomerationseffekte durch gezielte Anordnung von Einzelhandelsangeboten in möglichst großer Anzahl und Fläche sowie Leerstandsmanagement.

Allerdings stellen die oft sehr differenzierten bzw. zum Teil unübersichtlichen Eigentumsverhältnisse gepaart mit möglichem Desinteresse für den Standort eine große Handlungsschwelle im städtischen Kontext dar. Dem entsprechend ist zu prüfen, wie eine gezielt etablierte Form der Kooperation (im Sinne eines Centermanagements z. B. in Anlehnung an Immobilien- und Standortgemeinschaften, BIDs etc.) zur Verbesserungen der Zusammenarbeit zwischen Einzelhändlern und Immobilieneigentümern führen kann.

Eine zusätzliche Herausforderung für den innerstädtischen Einzelhandel kommt in jüngster Vergangenheit durch die stark wachsende Konkurrenz des Online-Handels hinzu. Aktuell geht der deutsche Einzelhandelsverband (HDE) davon aus, dass in Deutschland im Jahr 2025 rund 27\% vom Gesamteinzelhandelsumsatz durch den Online-Vertrieb generiert werden (REINK 2014, S. 15). Diese Tendenzen sind für zukünftige Handlungsoptionen zu berücksichtigen.

\subsection{Handlungsfeld Vernetzen und Steuern}

Unter der Maßgabe der Förderung partnerschaftlicher Zusammenarbeit fördert und unterstützt die Stadtentwicklungspolitik auf Bundes- und auf Landesebene die Eta- 
blierung von Standortkooperationen wie z. B. BID's, ISG's etc. Das Handlungsfeld Vernetzen und Steuern verweist einerseits auf die notwendige inhaltliche Basis und andererseits auf die organisatorische Ausgestaltung gemeinsamen Handelns, vor dem Hintergrund der Erkenntnisse eines professionellen Centermanagements in Shoppingcentern.

Die übergeordnete Zielstellung im Handlungsfeld Vernetzen und Steuern lautet dementsprechend:

\section{- Entwicklung und Etablierung eines neuen Bündnisses Innenstadt.}

Erfolgsfaktoren Shoppingcenter: Der Erfolg von Shoppingcentern hängt maßgeblich von einem professionellen Centermanagement ab. Dessen Aufgabenspektrum ist vielschichtig, es umfasst i. d. R:

- die Immobilienverwaltung,

- das Vermietungs- und Vertragsmanagement,

- das Flächenmanagement,

- die Marktforschung sowie

- das Marketing und die Beratung.

Die umfassende Handlungsfähigkeit des Centermanagements ist einerseits in der per Mietvertrag zugesprochenen Kompetenz hinsichtlich aller Aufgabenfelder des Centermanagements und andererseits in der finanziellen Ausstattung (durch die Pflichtmitgliedschaft und Beitragsentrichtung in der Werbegemeinschaft des Shoppingcenters) begründet. Die finanziellen Ressourcen fließen u. a. in einen Marketingfonds ein, der die Umsetzung einer gemeinsamen Marketingstrategie und Öffentlichkeitsarbeit ermöglicht.

Zudem basiert das wirksame Handeln des Centermanagements auf der zentralen Bündelung der verschiedenen Aufgabenfelder, so dass es möglich ist, inhaltliche Zusammenhänge und Wechselwirkungen zu erkennen sowie redundanten Arbeitsaufwand zu vermeiden, um daraus strategische Vorteile zu generieren.

Übertragung auf den städtischen Kontext: In Bezug auf die Arbeitsfelder des Centermanagements haben sich auch auf städtischer Ebene öffentlich initiierte oder privatwirtschaftlich organisierte Kooperationsformen wie z. B. Stadt- und Citymarketing, BID's oder ISG's herausgebildet. Besonders in Bezug auf das Standortmarketing, die Interessenvertretung der Einzelhändler sowie gemeinsame Gestaltungsfragen etc. leisten bestehende Standortkooperationen bereits einen wichtigen Beitrag zur innerstädtischen Standortaufwertung.

Allerdings bedarf es mit Blick auf die Problemlagen in Klein- und Mittelstädten und im Vergleich zu den Handlungsfeldern eines Shoppingcenter Managements teilw. umfangreicherer Handlungsansätze. Dabei müssen die Agglomerationspotenziale sowohl für die Kunden, die Einzelhändler als auch die Stadtentwicklung deutlich gemacht und erschlossen werden, wie z. B. durch:

- ein abgestimmtes Mietflächen- und Immobilienmanagement (inkl. Investitionskoordination),

- die Steuerung eines ausgewogenen Branchen- und Mietermixes sowie 
- eine kontinuierliche Unterstützung der Profilierung der involvierten Einzelhändler.

Dazu bedarf es der kooperativen Entwicklung einer gemeinsam abgestimmten Standortmarketing- und -managementstrategie (Mossig und Dorenkamp 2010). Daraus leiten sich die Unterziele 1 und 2 ab:

- Entwicklung und Etablierung eines neuen Bündnisses Innenstadt, welches in Anlehnung an das Centermanagement oder an bestehende Instrumente wie BID's, umfassende Handlungsspielräume erschließt.

- Entwicklung eines gemeinsam abgestimmten kooperativen Standortmarketing- und -managements durch Bündelung aller Akteure der Stadt- und Einzelhandelsentwicklung.

\subsection{Handlungsfeld: Beobachten (Monitoring)}

Das Handlungsfeld Beobachten bündelt die Inhalte sowie Zielstellungen der vorangegangenen Handlungsfelder im Sinne der Abschätzung sich vollziehender Veränderungen der Rahmenbedingungen sowie der Erfolgskontrolle mit Bezug auf die übergeordneten Ziele der Stadtentwicklungsplanung und ist somit als Informationsund Strategiebasis zu verstehen.

Das Ziel im Handlungsfeld Beobachten lautet:

- Schaffung der Grundlage für die aktive Steuerung der Entwicklung des Einzelhandelsstandortes Innenstadt durch Beobachten, Bewerten und Reflektieren.

Erfolgsfaktoren Shoppingcenter: Eine kontinuierliche Marktbeobachtung (auf lokaler und globaler Ebene) ist wesentlicher Bestandteil der Aufgaben des Centermanagements und liefert die entscheidenden Informationen für eine stete Anpassung des Shoppingcenter Betriebs/Marketing an sich verändernde Rahmenbedingungen (z. B. Sortimentspolitik, Preispolitik, Servicepolitik sowie Kommunikationspolitik) und eine begleitende Erfolgskontrolle (SIEDE 2005, S. 57). Neben den Umsatzzahlen, welche kontinuierlich von den Mietern des Shoppingcenters an das Centermanagement geliefert werden, sind u. a. Informationen zu den Passantenfrequenzen, zur Verweildauer und zur Besuchshäufigkeit von Interesse.

Übertragung auf den städtischen Kontext: Auch im städtischen Kontext bildet die Beobachtung einzelhandelsrelevanter Kriterien eine wichtige Grundlage für die Entwicklung sowie die kontinuierliche Anpassung eines strategischen Einzelhandelskonzeptes an die Rahmenbedingungen des Innenstadtstandortes und die Erfolgskontrolle umgesetzter Maßnahmen.

Die zu beobachtenden Kernkriterien sollten neben der wirtschaftlichen Performance, durch Veränderungen:

- der Verkaufsflächengröße,

- der 1a-Lage,

- der Passantenfrequenzen und dem Laufverhalten der Kunden, 
- dem Branchen- und Sortimentsmix,

- der Flächenangebote,

- der Mietpreise sowie

- der Einzelhandelszentralität.

auch die demografische sowie die soziokulturelle Entwicklung des Standortes Innenstadt abbilden. Darüber hinaus sind Informationen einzelhandelsbeeinflussender Bereiche wie z. B. dem Tourismus zu berücksichtigen.

Zusammenfassend resultiert daraus der folgende Zielkatalog:

Dabei ist zu beachten, dass dieser einerseits keine Vorgehensweise impliziert, sondern lediglich Handlungserfordernisse resultierend auf dem identifizierten Erfolgsfaktorenset (vgl. Tab. 1) formuliert, demnach sind mögliche Prozesszusammenhänge und iterative Vorgehensweisen nicht Bestandteil des Zielkatalogs. Andererseits wird mit der Formulierung der folgenden Zielstellungen die Perspektive der Stadtentwicklungsplanung eingenommen, wodurch in erster Linie die Kommune als Zielgruppe im Fokus steht.

\section{5 Überprüfung der Umsetzbarkeit in der Praxis}

Der Aspekt der Übertragbarkeit/Generalisierbarkeit dieser Erkenntnisse wurde anhand einer konkreten Anwendung des Zielkatalogs in der Stadt Torgau überprüft, wodurch die eingangs aufgestellte Hypothese bestätigt werden konnte. In einem ersten Schritt erfolgte dabei die Operationalisierung der übergeordnet definierten Zielstellungen des Zielkatalogs auf lokaler Ebene, wobei anhand der dabei identifizierten Bewertungskriterien eine Stärken-Schwächen-Analyse durchgeführt wurde. Der zweite Schritt diente darauf aufbauend der Entwicklung einer idealtypischen Innenstadtkonzeption Einzelhandel sowie der Diskussion dieser mit davon betroffenen öffentlichen und privaten Akteuren. ${ }^{16}$

Dieses Vorgehen zeigte, dass es nicht nur grundsätzlich möglich ist, die im Zielkatalog formulierten Anforderungen in die Praxis zu übertragen, sondern diese gewinnbringend für die Stadtentwicklungsplanung zu nutzen. Denn die Übertragung der Zielstellungen sowie deren Operationalisierung lieferte einerseits wesentliche inhaltliche Anstöße für die planerische Entwicklung einer idealtypischen Innenstadtkonzeption verdeutlichte allerdings andererseits Schwachstellen auf dem Weg einer möglichen Umsetzung, wie bspw.:

Handlungsfeld: Gestalten und Verorten:

- Schwache Wahrnehmung und Nutzung der Qualität der städtebaulichen, architektonischen sowie funktionalen Bezüge einer historisch gewachsenen Innenstadt als zentrales Alleinstellungsmerkmal für die Einzelhandelsfunktion.

\footnotetext{
${ }^{16}$ Die Anwendung des Planungsinstrumentariums wurde an dieser Stelle nicht in die Betrachtungen mit einbezogen, da es in der Dissertation um die grundsätzlich theoretische Überprüfung der Übertragbarkeit des Zielkatalogs ging. Die konkrete Ausgestaltung der weiteren Vorgehensweise wäre individuell bezogen auf konkrete Standorte in weiteren Arbeitsschritten zu klären. Da die Übertragung des Zielkatalogs lediglich in einer Stadt, Torgau, durchgeführt wurde können an dieser Stelle keine Erfolgskriterien bezüglich der Übertragbarkeit formuliert werden. Dafür wäre eine breiter angelegte Empirie notwendig.
} 
Tab. 2 Zielkatalog innerstädtische Einzelhandelsentwicklung (eigene Darstellung)

Zielkatalog innerstädtische Einzelhandelsentwicklung

Handlungsfeld Gestalten und Verorten

Übergeordnetes Ziel: Entwicklung eines städtebaulich und architektonisch abgestimmten Einzelhandelsstandortes Innenstadt mit engen funktionalen Wechselbezügen

Unterziel 1: Qualitätssicherung der städtebaulichen, architektonischen und funktionalen Gestaltung des EZH-Standortes Innenstadt (Stichwort: Urbane Differenz)

Unterziel 2: Gestaltung eines städtebaulich, stadträumlich und funktional kohärenten „Rundlaufs“

Unterziel 3: Gestaltung und Aufwertung des öffentlichen Raums sowie Erhaltung seiner öffentlichen Funktion

\section{Handlungsfeld Handeln und Vermarkten}

Übergeordnetes Ziel: Anpassung des innerstädtischen Einzelhandelsangebotes an eine sich verändernde Zielgruppe und an veränderte Kundenbedürfnisse sowie Sicherung einer größtmöglichen Angebotsvielfalt und -tiefe mit Blick auf die Gesamtagglomeration Innenstadt

Unterziel 1: Anpassung und Abstimmung des innerstädtischen Einzelhandelsangebotes an veränderte Zielgruppen und steigende Convenience-, Service- und Erlebnisbedürfnisse, wie z. B. Emotionalisierung des Einkaufens (unter Berücksichtigung von Konkurrenzstandorten)

Unterziel 2: Erzeugung möglichst großer Synergie- und Agglomerationseffekte durch gezielte Anordnung von Einzelhandelsangeboten in möglichst großer Anzahl und Fläche (Gleichgewicht zwischen frequenzstarken Ankermietern und Sortimentsergänzenden EZH-Angeboten) sowie Leerstandsmanagement

Handlungsfeld Vernetzen und Steuern

Übergeordnetes Ziel: Entwicklung und Etablierung eines neuen Bündnisses Innenstadt.

Unterziel 1: Entwicklung und Etablierung eines neuen Bündnisses Innenstadt, welches in Anlehnung an das Centermanagement oder an bestehende Instrumente wie BID's, umfassende Handlungsspielräume erschließt

Unterziel 2: Entwicklung eines gemeinsam abgestimmten kooperativen Standortmarketing- und -managements durch Bündelung aller Akteure der Stadt- und Einzelhandelsentwicklung (z. B. im Rahmen eines innerstädtischen Einzelhandelskonzeptes)

Handlungsfeld Beobachten

Ziel: Schaffung der Grundlage für die aktive Steuerung der Entwicklung des Einzelhandelsstandortes Innenstadt durch Beobachten, Bewerten und Reflektieren

- Dichte innerstädtische Baustrukturen bieten wenig Entwicklungspotenzial für neue Einzelhandelsansiedlungen.

- $\mathrm{Zu}$ geringe finanzielle Ausstattung für notwendige Investitionen im öffentlichen Raum.

\section{Handlungsfeld: Handeln und Vermarkten:}

- Die Uneinigkeit sowie die Nicht-Organisation der involvierten Akteure, stellen den Ausgangspunkt für verschiedene Problemlagen dar.

- Die Entwicklung eines abgestimmten, kopplungsfördernden Einzelhandelsangebotes erfordert einen hohen Moderations- und Managementaufwand zwischen den Einzelhändlern.

\section{Handlungsfeld: Vernetzen und Steuern}

- Die gegenseitigen Erwartungshaltungen der Akteure der Stadtverwaltung sowie der Einzelhändler erschweren die Etablierung einer neutralen, zentralistisch agierenden Innenstadtkoordination. 
- Ein hoher Grad an Einzeleigentümern in unterschiedlichsten finanziellen Situationen erschwert ein abgestimmtes Handeln.

- Die Erschließung von Städtebaufördermitteln aufgrund sinkender Fördermittelsätze und fehlender personeller Ausstattung der Stadtverwaltungen wird zunehmend erschwert.

\section{Handlungsfeld: Beobachten (Monitoring)}

- Die regelmäßige Fortschreibung eines Monitorings erfordert einen hohen Personalaufwand.

Auch wenn die benannten Schwächen eine große Herausforderung für die innerstädtische Einzelhandelsentwicklung darstellen, zeigen die Prüfergebnisse allerdings, dass vor allem im aktiven Umgang mit der Problematik der Akteurs- und Handlungsheterogenität sowie der Förderung von Selbststeuerungsprozessen im Rahmen der Stadtentwicklungsplanung der Schlüssel zur Lösung liegt (Baumgart und Rüdiger 2010, S. 196).

Im inhaltlichen Rückschluss auf den Zielkatalog innerstädtische Einzelhandelsentwicklung bedeutet das, dass das Handlungsfeld Vernetzen und Steuern in Bezug auf seine Bedeutung für das lokale Handeln an erster Stelle neben dem Handlungsfeld Gestalten und Verorten stehen muss. Somit hängt der Erfolg des vorgeschlagenen Vorgehens letztendlich von der Verknüpfung von konsensual entwickelten Strategien, unter maßgeblicher Berücksichtigung der städtebaulichen, architektonischen sowie funktionalen Qualitäten historisch gewachsener Innenstädte, und dem abgestimmten kooperativen Handeln der Akteure vor Ort ab.

Damit richtet sich das entwickelte Instrumentarium in erster Linie an die Stadtverwaltung von Klein- und Mittelstädten mit dem Ziel, das Bewusstsein für ganzheitliche, integrative Planungsansätze zu stärken sowie die Stadt als gebauten Raum und funktionales Netzwerk als wesentliches Potenzial für zukünftige Entwicklungen zu verstehen.

Elektronische Quelle (Bundesfinanzministerium, 2012) http:/www.bundesfinanzministerium.de/ nn_137870/DE/BMF_Startseite/Publikationen/Monatsbericht_des_BMF/2011/08/analysen-und-berichte/b02-gemeindefinanzkommission/Gemeindefinanzkommission.html\#2, abgerufen am 27.01.2012.

Open Access Dieser Artikel unterliegt den Bedingungen der Creative Commons Attribution License. Dadurch sind die Nutzung, Verteilung, und Reproduktion erlaubt, sofern der/die Originalautor/en und die Quelle angegeben sind.

\section{Literatur}

Adam, B. (2010). Struktur und Funktion der Mittelstädte in Stadtregionen - Was ist das Besondere? unveröffentlichtes Manuskript, Vortrag März 2010, Institut für Raumplanung Universität Dortmund.

Bastian, A. (1999). Erfolgsfaktoren von Einkaufszentren. Ansätze zur kundengerichteten Profilierung, Betriebswirtschaftlicher Verlag Dr. Th. Wiesbaden: Gabler GmbH und Deutscher Universitätsverlag $\mathrm{GmbH}$. 
Baumgart, S. (2004). Einführung in das Forschungsthema „Klein- und Mittelstädte“. In S. Baumgart, J. Flacke, C. Grüger, P. Lütke, \& A. Rüdiger (Hrsg.), Kleine und mittlere Städte - Blaupausen der Großstadt, Dokumentation des Expertenkolloquiums am 29. April 2004 in Dortmund (S. 7-10). Dortmund: Fakultät Raumplanung Universität Dortmund.

Baumgart, S., \& Rüdiger, A. (2010). Mittelstädte im demographischen Wandel - Herausforderungen für die strategische Stadtentwicklung. In B. Schmidt-Lauber (Hrsg.), Mittelstadt: Urbanes Leben jenseits der Metropole (S. 189-204). Frankfurt a. M.: Campus Verlag GmbH.

Besemer, S. (2004). Shopping-Center der Zukunft. Planung und Gestaltung. Wiesbaden: Deutscher Universitätsverlag, GWV Fachverlage GmbH.

Bolz, N. (2002). Das konsumistische Manifest. München: Wilhelm Fink Verlag.

Brune, W. (2014). 50 Jahre Shopping-Center-Erfahrung - was bringt die Zukunft?. In: Falk \& B; Falk, M. (Hrsg.), Shopping Center Future (S. 142-147). Starnberg: Stolz Druck GmbH, Mitterfels.

Dach, C. (2002). Internet Shopping versus stationärer Handel, Schriften zur Handelsforschung Bd. 98, Institut für Handelsforschung Köln. Stuttgart: W. Kohlhammer GmbH.

Deutsche Hypothekenbank/Immobilien Research (Hrsg.). (2009). Einzelhandelsimmobilien - Trends auf der Angebotsseite, Deutsche Hypothekenbank/Immobilien Research.

Esser, B. (2002). Smart Shopping. eine theoretische und empirische Analyse des preis-leistungsorientierten Einkaufsverhaltens von Konsumenten, Dissertation, Betreut von Prof. Dr. Richard Köhler. Köln: Universität zu Köln.

Falk, B. (2014). Zur Entwicklung der Shopping-Center: 1925-1950-1964-2014 -?. In B. Falk \& M. T. Falk (Hrsg.), Shopping Center Future (S. 105-117). Starnberg: Stolz Druck GmbH, Mitterfels.

Flacke, J. (2004). Definitionen, Merkmale und Typologien von Klein- und Mittelstädten. In S. Baumgart, J. Flacke, C. Grüger, P. Lütke, \& A. Rüdiger (Hrsg.), Klein- und Mittelstädte - Verkleinerte Blaupausen der Großstadt? Dokumentation des Expertenkolloquiums am 29. April 2004 an der Universität Dortmund (SRPapers, Nr. 1) (S. 27-34). Dortmund: Fachgebiet Stadt- und Regionalplanung Fakultät Raumplanung, Universität Dortmund.

Funck, D., Gutknecht, K., \& Stumpf, J. (2007). Mittelmaß verboten! Die Zukunft des mittelständischen Facheinzelhandels liegt in der herausragenden Positionierung und Profilierung. In V. Trommsdorff (Hrsg.), Handelsforschung 2007 (S. 30-42). Stuttgart: W. Kohlhammer GmbH.

Gatzweiler, H.-P. (2010). Städtebauliche Herausforderungen in ländlichen Räumen. in Bundesministerium für Verkehr, Bau und Stadtentwicklung (Hrsg.): Starke Mittelstädte: Städtebauförderung in ländlichen Räumen, Kongress 22. Juni 2010 in Berlin, Kongressdokumentation, Oldenburg, S. 17-23.

Germelmann, C. C., \& Neder, P. (2012). Shopping-Center. In J. Zentes, B. Swoboda, D. Morschett, \& H. Schramm-Klein (Hrsg.), Handbuch Handel. Strategien - Perspektiven - Internationaler Wettbewerb (S. 329-349). Wiesbaden: Springer Fachmedien.

Hansen, U. (1990). Absatz- und Beschaffungsmarketing des Einzelhandels. Göttingen: Vandenhoeck \& Ruprecht.

Hatzfeld, U. (1998). Malls und Mega-Malls. Globale Investitionsstrategien und lokale Verträglichkeit. In G. Hennings \& S. Müller (Hrsg.), Kunstwelten. Künstliche Erlebniswelten und Planung, Informationskreis für Raumplanung e. V. (Dortmunder Beiträge zur Raumplanung, 85) (S. 32-50) Dortmund: Institut für Raumplanung Universität Dortmund Fakultät Raumplanung.

Heinritz, G., Popp, M., \& Pesch, F. et al (2003). Forschungsbericht Innerstädtische Einkaufszentren, Oberste Baubehörde im Bayrischen Staatsministerium des Innern, München: Oberste Baubehörde im Bayerischen Staatsministerium des Innern.

Hirsch, J. (2011). Quo vadis, Einzelhandel in Mittelstädten? - Kommunale Umfrage in Mittelstädten zur Bedeutung des Handels für eine nachhaltige Stadtentwicklung, Competence Center Retail Property (CCRP). Regensburg: Universität Regensburg.

Just, T. (2014), Ökonomische Bestimmungsfaktoren für Shopping-Center. In Falk, B. \& Falk, M. (Hrsg.), Shopping Center Future (S. 34-48). Starnberg.

Kabisch, S., Bernt, M., \& Peter, A. (2004). Stadtumbau unter Schrumpfungsbedingungen. Wiesbaden: VS Verlag für Sozialwissenschaften/GWV Fachverlage GmbH.

Koprian, H. (2014). Innenstadtgalerien und Einzelhandel - Ein Blick zurück und nach vorne. In Falk B. \& Falk M. (Hrsg.), Shopping Center Future (S. 137-141). Starnberg.

Martin, A. (2003). Entscheidungsprogramme der Branchenmixpolitik für regionale Shoppingcenter im Kontext der strategischen Marketingplanung. Frankfurt a. M.: Peter Lang GmbH.

Mikunda, C. (2009). Marketing spüren: Willkommen am dritten Ort. München: Redline Verlag.

Monheim, R., \& Heller, J. (2011). Die Innenstadt von Leipzig aus Sicht ihrer Besucher - attraktiv durch urbane Vielfalt. Bayreuth: Verlag Naturwissenschaftliche Gesellschaft Bayreuth. 
Mossig, I., \& Dorenkamp, A. (2010). Shopping-Malls und Business Improvement Districts als Instrumente zur Belebung innerstädtischer Geschäftszentren? Das Beispiel der Stadt Gießen. In Beiträge zur Wirtschaftsgeographie und Regionalentwicklung, Bd. 2. URL: http://nbn-resolving.de/urn:nbn:d e:gbv:46-00102045-16. Zugegriffen: 12. Aug. 2014.

Müller, J., \& Stein, C. (2008). Smart Shopping im Einzelhandel, Reihe Marketing-Praxis. Bd. 2, Lohmar, Köln: Josef EUL Verlag GmbH.

Ortner, H. (2014). Shopping-Center-Entwicklung in Mittelstädten bis 2020. In Falk B. \& Falk M. (Hrsg.), Shopping Center Future (S. 148-161). Starnberg.

Purper, G. (2007). Die Betriebsformen des Einzelhandels aus Konsumentenperspektive. Wiesbaden: Deutscher Universitäts-Verlag/GWV Fachverlage GmbH.

Reink, M. (2014). Der Einzelhandel - Situation und Herausforderungen, unveröffentlichtes Manuskript, Tagung des Wissensnetzwerks Stadt und Handel am 24./25.04.2014, Hamburg.

Siebel, W. (2007). Vom Wandel des öffentlichen Raums. In J. Wehrheim (Hrsg.), Shoppingmalls. Interdisziplinäre Betrachtungen eines neuen Raumtyps (S. 77-94). Wiesbaden: VS Verlag für Sozialwissenschaften/GWV Fachverlage GmbH.

Siede, H. (2005). Center-Management in Einkaufszentren, Diplomarbeit, Hochschule für angewandte Wissenschaften Hamburg, Fachbereich Ökotrophologie, Studiengang Ökotrophologie, Hamburg.

Sturm, V. (2006). Erfolgsfaktoren der Revitalisierung von Shopping-Centern. Köln: Immobilien Informationsverlag Rudolf Müller GmbH \& Co. KG.

Urban Land Institut (1993). Dollars \& Cents of Shoppingcenters in the TOP 20 Metropolitan Areas. A Special Report, Washington, D.C.

Wengler, T. (2005). Auswirkungen des Internet-Handels auf Shopping-Center, Dissertation. Norderstedt: Books on Demand GmbH. 